\title{
Supplemental Figure 1.
}

Example of manual annotation of a false positive due to methylation. A. Spectrum labeled with ion peaks corresponding to alternative allele of nsSNP rs11543322. B. Same spectrum labeled with ion peaks corresponding to the reference allele of rs 11543322 containing a methylated glutamate at position 10. Note additional assignment of 5 ion peaks from mass fragments of a methylated reference allele versus the alternative SNP allele (arrows). 